\title{
Bronchoalveolar lavage in COPD: fluid recovery correlates with the degree of emphysema
}

\author{
J.M. LöfdahI*, K. Cederlund" ${ }^{\#}$ L. Nathell", A. Eklund* and C.M. Sköld*
}

ABSTRACT: Bronchoscopy with bronchoalveolar lavage (BAL) is an important research tool for assessing airway inflammation in a variety of inflammatory lung diseases. In chronic obstructive pulmonary disease (COPD), BAL recovery is often low, making analysis of the recovered fluid difficult to interpret. The present authors hypothesised that the degree of emphysema may predict BAL recovery.

A total of 20 COPD patients (mean age 57 yrs, range 49-69) with a median (interquartile range) forced expiratory volume in one second (FEV1) of 51 (33-69)\% predicted underwent BAL. Matched "healthy" smokers and nonsmokers served as controls. Emphysema index in COPD patients was calculated on computed tomography scan as the percentage of the right lung with pixels $<-950$ Hounsfield units. The carbon monoxide diffusing capacity of the lung (DL,CO) was determined by the single-breath method.

COPD patients had lower BAL recovery than controls. COPD patients with an emphysema index $<1$ had higher BAL recovery than patients with an emphysema index $>1$. BAL recovery correlated negatively to emphysema index and positively to DL,Co. However, no correlation was found between recovery and FEV1.

In conclusion, the extent of emphysema evaluated by computed tomography-scan index and carbon monoxide diffusing capacity of the lung may predict a low bronchoalveolar lavage recovery in chronic obstructive pulmonary disease patients. These parameters may, therefore, be useful when chronic obstructive pulmonary disease patients are selected for bronchoscopy with bronchoalveloar lavage. The present study underlines the importance of careful phenotyping of chronic obstructive pulmonary disease patients.

KEYWORDS: Bronchoalveloar lavage, bronchoalveolar lavage fluid, bronchoscopy, carbon monoxide diffusing capacity of the lung, chronic obstructive pulmonary disease, pulmonary emphysema

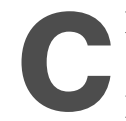

hronic obstructive pulmonary disease (COPD) is a heterogeneous disease comprising of a variety of manifestations such as bronchitis, bronchiolitis and emphysema $[1,2]$. Inflammatory changes are present in proximal airways [3] as well as in peripheral airways and lung parenchyma [4]. Studies on lungs removed during surgery and post mortem [5], indicate that the major site of airways obstruction is in the bronchioles $[6,7]$. However, emphysema may also contribute to airflow limitation [8]. Thus, bronchiolitis and emphysema can both be present in COPD patients and their relative contributions to airflow limitation vary from one patient to another.

Mechanisms underlying the development of airways obstruction are not fully understood. In this context, bronchoalveolar lavage (BAL) is a useful and safe research method for sampling cells and mediators from the lower respiratory tract [9]. However, in COPD the recovered lavage volumes are often low [10, 11] and the BAL findings can, therefore, be difficult to interpret. In clinical guidelines it was reported that BAL recovery is reduced to $10-40 \%$ of that instilled [9]. Furthermore, a low BAL recovery may predominantly reflect the larger airways [12], which is a lung compartment that is not a major contributor to airways obstruction in COPD. The present authors hypothesised that the degree of emphysema may influence BAL recovery in COPD patients. To test this hypothesis, the current authors examined if measures of emphysema could predict BAL recovery in COPD patients. Specifically, lung function and radiological parameters were

\section{AFFILIATIONS}

*Dept of Medicine, Division of Respiratory Medicine, Karolinska University Hospital, Solna

\#Dept of Radiology, Karolinska University Hospital, Huddinge, and - Personal Injury Prevention Section, Dept of Clinical Neuroscience, Karolinska Institutet, Stockholm, Sweden.

CORRESPONDENCE

J.M. Löfdahl

Dept of Respiratory Medicine Karolinska University Hospital S-171 76 Stockholm Sweden

Fax: 46332998

E-mail: magnus.lofdahl@

karolinska.se

Received:

March 182004

Accepted after revision:

October 022004

\section{SUPPORT STATEMENT}

The study was supported by grants from the Swedish Heart Lung Foundation, King Oscar II Jubilee fund, the Cancer and Allergy Foundation, the Swedish Foundation for Health Care Sciences and Allergy Research, Karolinska Institutet and Boehringer-Ingelheim/Pfizer. 
determined in a group of patients with moderate-to-severe COPD. These patients underwent bronchoscopy with BAL. "Healthy" smokers and nonsmokers served as controls.

\section{PATIENTS AND METHODS}

\section{Patients and control subjects}

A total of 20 patients (mean age 57 yrs, range 49-69) with moderate-to-severe COPD were studied. All patients had a post-bronchodilator forced expiratory volume in one second (FEV1)/vital capacity (VC) $<70 \%$ and a post-bronchodilator FEV1 $<70 \%$ predicted. The COPD patients had a smoking history of $>10$ pack yrs. Of the study patients, 16 were current smokers and four had quit smoking. Two groups of agematched controls were included: one group of asymptomatic smokers with normal spirometry values and one group of "healthy" nonsmokers. Patients and control subjects characteristics are given in table 1 . All subjects in the two control groups had a normal chest radiograph. The smoking history measured as pack-yrs did not differ between the COPD patients and the "healthy" smokers. The COPD patients had a higher $(p<0.05)$ median reversibility of FEV1 than the two control groups.

None of the patients or control subjects had medicated with inhaled or orally administrated steroids 3 months prior to the study. The COPD patients were in a stable clinical condition defined as the absence of exacerbation for the last 3 months. All patients and control subjects gave their informed consent to participate, and the study protocol had the approval of the local ethics committee (Ethical Research Committee, Karolinska Institute, Stockholm, Sweden).

\section{Bronchoscopy and BAL}

Bronchoscopy was performed on an outpatient basis, following overnight fasting. After pre-medication with morphine-hyoscine i.m. $45 \mathrm{~min}$ prior to the investigation, the bronchoscope (Olympus F Type P30, Olympus Optical Co. Ltd, Tokyo, Japan) was inserted nasally after topical anaesthesia with lignocaine (Xylocain ${ }^{\circledR} ;$ AstraZeneca, Södertälje, Sweden). The participants did not receive any other medication before the bronchoscopy.
BAL was performed by wedging the bronchoscope in a subsegment of the middle lobe. In one of the patients, due to difficulties in wedging the bronchoscope in the middle lobe, BAL was performed in one of the basal subsegments of the right lower lobe. Five aliquots of $50 \mathrm{~mL}$ of sterile PBS at $37^{\circ} \mathrm{C}$ were instilled and gently suctioned back with a negative pressure of $-40--50 \mathrm{mmHg}$. Dwell time was kept to a minimum as recommended by the European Respiratory Society (ERS) task force [13]. In order to avoid collapse of peripheral airways, the pressure was adjusted to between -10$-20 \mathrm{mmHg}$ if BAL recovery appeared to be poor. BAL was interrupted if the recovery, after 150-200 mL of instilled fluid, was $<35 \mathrm{~mL}$, or if persistent cough or desaturation occurred ( $<90 \%$ oxygen saturation despite appropriate oxygen supplement). The fluid was collected in a silicone treated bottle kept on ice, which was immediately transported to the laboratory.

\section{Processing of BAL fluid}

BAL fluid was filtered from mucus with a Dacron net (Millipore, Cork, Ireland) and the volume of recovered fluid was measured. Recovery was expressed both as absolute volume and as percentage of instilled fluid. Cells were pelleted by centrifugation at $400 \times \mathrm{g}, 4^{\circ} \mathrm{C}$, for $10 \mathrm{~min}$ and the supernatants were removed. The cell pellets were resuspended in RPMI medium and total numbers of cells were counted in a Bürker chamber (Marienfeld, Germany).

\section{Radiological examination}

The patients underwent examination with computed tomography (CT) using a Siemens Somatom Plus $S$ scanner (Siemens, Erlangen, Germany). The spiral CT was performed with $10 \mathrm{~mm}$ slice thickness and pitch 1.0-1.2 depending on the length of the lungs. The scanning direction was cranio-caudal. All examinations were performed in maximal inspiration and without i.v. contrast enhancement. For spiral CT the tube current was $210 \mathrm{~mA}$, exposure time (rotation time) $1 \mathrm{~s}$ and the voltage $120 \mathrm{kV}$. The images were reconstructed with a highspatial frequency algorithm (defined for the actual scanner as AB 7541). High-resolution computed tomography (HRCT) was also performed for visual evaluation.

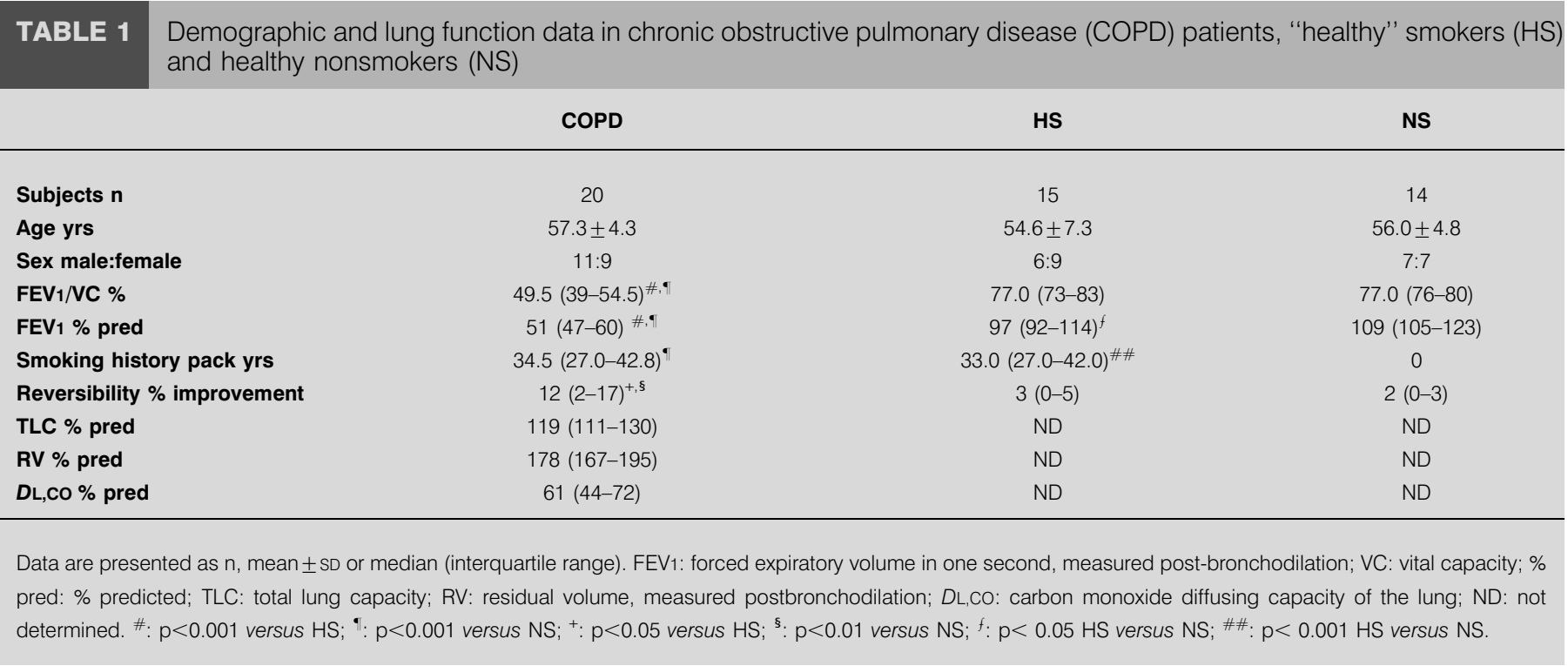


The severity of emphysema was defined as emphysema index, which is an objective quantification assessing the extent of emphysema and eliminating observer variability. These types of indexes have been validated by comparing quantitative CT data with histopathology specimens [14-16]. The emphysema index was defined as the relative area of the CT image occupied by pixels between -1024 and -950 Hounsfield units. For the assessment of emphysema index, spiral CT scans with $10 \mathrm{~mm}$ slice thickness were used. The images were analysed with Siemens Pulmo CT software, a semi-automatic imageprocessing program that enables detection and outlining of the lung margins. The software subsequently presents histograms of attenuation values and calculates the percentage of the lung area with pixels within the predefined interval of Hounsfield units. The degree of emphysema can thereby be expressed as an emphysema index. Thus, emphysema index expresses the relative area of the CT-scan slices with pixels below the defined threshold [17]. Emphysema index was calculated in 18 patients. Due to technical reasons, calculations were not done in two patients. In addition to emphysema index assessments, all HRCT scans were evaluated visually by an experienced radiologist. Particular attention was given to evaluation regarding occurrence of emphysema.

\section{Lung function tests}

Expiratory volumes were measured with a dynamic spirometer (Vitalograph ${ }^{\circledR}$, Buckingham, UK) in a standardised manner. Both forced vital capacity (FVC) and VC were determined. Reversibility of airways obstruction was tested by measuring FEV1 10 min after inhalation of two doses of $0.5 \mathrm{mg}$ terbutalin (Bricanyl ${ }^{\circledR}$ Turbuhaler ${ }^{\circledR}$; AstraZeneca). Reversibility was calculated as the percentage improvement of FEV1. Thoracic gas volume at end-tidal expiration was measured in a constant volume body plethysmograph by panting at slow frequency against a closed shutter. The carbon monoxide diffusing capacity of the lung $(D \mathrm{~L}, \mathrm{CO})$ was measured by the single-breath method. All the above parameters were expressed as percentages of the predicted values according to the respective European Coal and Steel Community references [18-22].

\section{Statistical methods and data management}

Statistical comparisons to test differences between the two groups were made by use of the Mann-Whitney U-test with continuity correction. In order to test differences between three groups, Kruskal-Wallis ANOVA and median test were used. The prognostic power of the different variables was compared by the use of stepwise regression analysis. In addition, descriptive statistics and graphical methods were used to characterise the data. The study employs multiple hypotheses testing, where each hypothesis was analysed separately and the existence of patterns in and the consistency of the results were considered in the analysis. The 5, 1 and $0.1 \%$ levels of significance were considered. In the case of a statistically significant result the p-value has been given.

\section{RESULTS}

\section{$B A L$ recovery in COPD patients and control subjects}

The recovered BAL fluid, expressed as volume $(\mathrm{mL})$ as well as recovered proportion $(\%)$, was significantly ( $p<0.001$ for both) lower in the COPD group compared to both control groups (fig. 1). Furthermore, BAL recovery was lower $(\mathrm{p}<0.05)$ in "healthy" smokers than in nonsmokers. The total cell yield was highest $(p<0.01)$ in the group of "healthy" smokers, whereas the total cell yield in the COPD group was higher $(p<0.05)$ than in nonsmokers. The cell concentration in the nonsmoking group was significantly lower than in the COPD group $(\mathrm{p}<0.001)$ and "healthy" smokers group $(\mathrm{p}<0.001)$, but the concentration did not differ between the COPD and the "healthy" smokers group.

In 10 of the 20 examined COPD patients, a complete BAL was performed using $250 \mathrm{~mL}$ of instilled fluid. In five of the patients, BAL was interrupted due to low recovery $(<35 \mathrm{~mL})$. In this subgroup of five patients, the mean instilled volume was $167 \mathrm{~mL}$ (range 150-200 $\mathrm{mL}$ ) and the mean recovery was $12.4 \%$ (range $7.3-18 \%$ ). In five additional COPD patients, BAL was interrupted due to persistent cough or desaturation. The mean instilled volume for these five patients was $190 \mathrm{~mL}$ (range 150-240 mL), and the mean recovery was 33.2\% (range $20-51 \%$ ). Thus, all COPD patients had a BAL of $\geqslant 150 \mathrm{~mL}$ instilled volume. The median instilled volume of BAL fluid in all COPD patients $(n=20)$ was significantly lower $(p<0.001$ for both) compared to both control groups. BAL characteristics are presented in table 2 .

\section{Computed tomography}

The number of spiral CT sections per lung varied from 17-24, depending on the length of the patients' lungs. The general degree of emphysema in each patient was approximated to the mean of emphysema indexes of the sections from one lung. Since BAL was performed in the right middle lobe, the mean of the right lung sections was used. The median emphysema index in the COPD patients was 1.59 (interquartile range (IQR) was $0.46-6.1$; fig. 2). Patients with an emphysema index $<1$ were generally without obvious radiological emphysema visual on $\mathrm{CT}$, whereas patients with an emphysema index $>1$ had visible emphysema in their CT scan (fig. 3). Hence, patients were divided into two groups: one group with a low

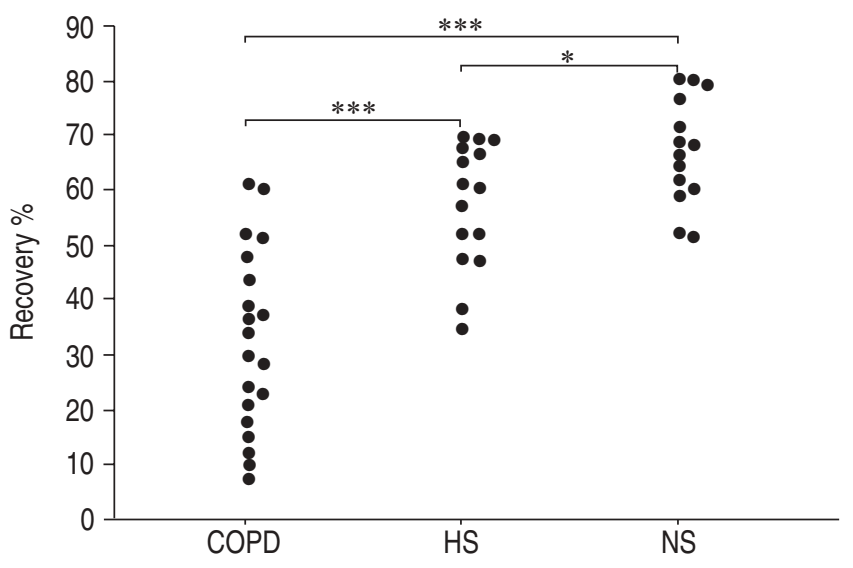

FIGURE 1. Bronchoalveolar lavage fluid recovery measured as a percentage of instilled fluid in subjects with chronic obstructive pulmonary disease (COPD), "healthy" smokers (HS) and nonsmokers (NS). Statistical comparison between groups was made using the Kruskal-Wallis ANOVA and median test, post hoc analysis by Mann-Whitney U-test with continuity correction. *: $p<0.05$; *** $\mathrm{p}<0.001$. 


\begin{tabular}{|c|c|c|c|}
\hline \multirow[t]{2}{*}{ TABLE 2} & acteristics in ch & onary disease & "healthy" smokers \\
\hline & COPD & HS & NS \\
\hline Subjects $\mathrm{n}$ & 20 & 15 & 14 \\
\hline Instilled volume $\mathrm{mL}$ & $245(168-250)^{\# \cdot \bullet}$ & $250(250-250)$ & $250(250-250)$ \\
\hline Recovery mL & $79(35-108)^{+, \S}$ & $146(119-168)^{\pi}$ & $169(150-192)$ \\
\hline Cell concentration $\times 10^{6} \cdot \mathrm{L}^{-1}$ & $331(108-620)^{\# \#}$ & $499(261-654)^{++}$ & $71(61-91)$ \\
\hline
\end{tabular}

Data are given as $n$ or median (interquartile range). ${ }^{\#:} p<0.01$ versus HS; ${ }^{\bullet}: p<0.01$ versus $N S ;{ }^{+}: p<0.001$ versus HS; ${ }^{\text {s. }} p<0.001$ versus NS; ${ }^{f}: p<0.05$ versus NS; $\#$ : $p<0.001$ versus $N S ;{ }^{~}{ }^{\circ}: p<0.05$ versus $N S ;{ }^{++}: p<0.001$ versus NS.

emphysema index, labelled EI $<1$, and one group with a high emphysema index, labelled EI $>1$. The EI $<1$ group $(n=7)$ had a median emphysema index of $0.43 \%$ (IQR $0.23-0.67$ ), whereas the EI $>1$ patients emphysema index was 3.9\% (1.9-7.5) and a median FEV1 of $51 \%$ predicted (IQR 48-61). The EI $>1$ group $(n=11)$ had a similar degree of airways obstruction measured as FEV1 \% pred (median 51\%, IQR 47-60). The emphysema index in the EI $>1$ group was $3.9 \%$ (IQR 1.9-7.5). The two groups had a similar degree of airway obstruction, measured both as FEV1 and FEV1/VC (table 3). However, DL,CO, which is thought to reflect the degree of emphysema [8, 23], was significantly lower in COPD patients with EI $>1$. In addition, both $\mathrm{DL}, \mathrm{CO}$ and $\mathrm{FEV} 1 / \mathrm{VC}$ correlated to emphysema index $(\mathrm{p}<0.05$ for both parameters, $\mathrm{r}=-0.50$ and -0.47 respectively).

\section{Correlations between BAL recovery and computed tomography}

The median BAL recovery in the EI $<1$ group $(n=7)$ was $51 \%$ (IQR 38-60), whereas the recovery in the EI $>1$ group $(n=11)$

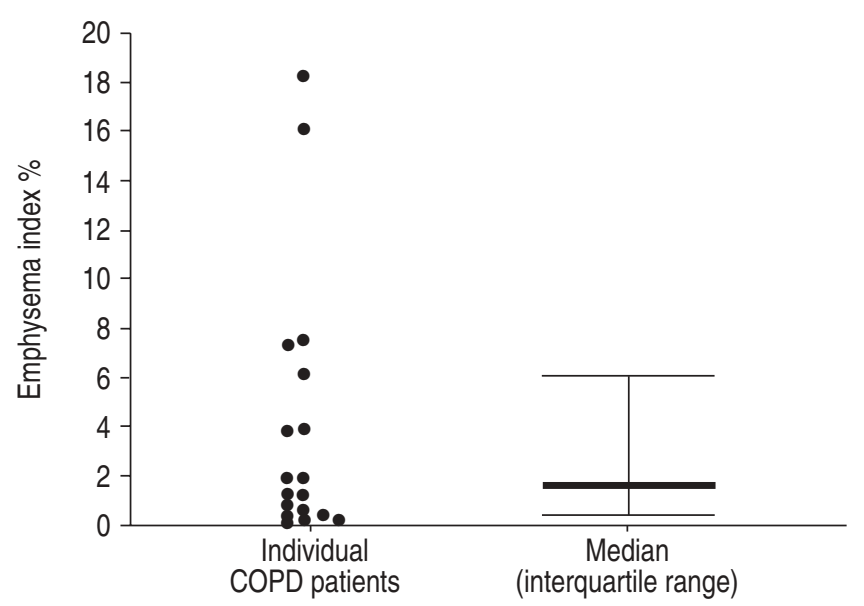

FIGURE 2. Emphysema indexes in chronic obstructive pulmonary disease (COPD) patients $(n=18)$, expressed as the percentage of the total computed tomography (CT) slice area of the right lung with pixels $<-950$ Hounsfield units. Two patients were omitted since they were not examined with $\mathrm{CT}$ scan due to technical reasons.
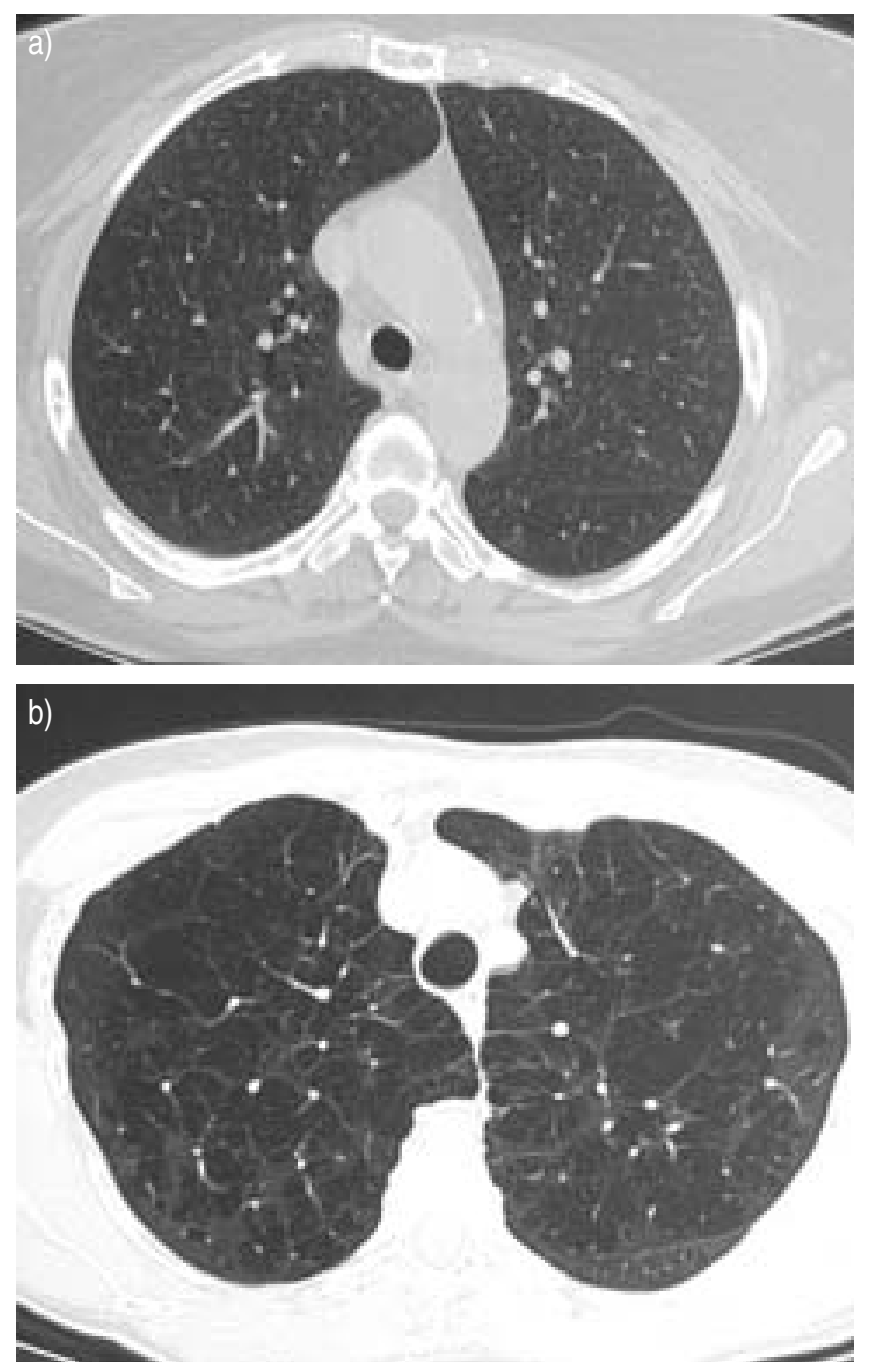

FIGURE 3. High-resolution computed tomography scans (slice thickness $2 \mathrm{~mm}$ ) of two patients included in the current study. Both have the same degree of airway obstruction, but their emphysema indexes and bronchoalveolar lavage (BAL) recovery differ. a) Forced expiratory volume in one second ( $\mathrm{FEV}_{1}$ ): $49 \%$ predicted; emphysema index: 0.23\%; BAL recovery: $51 \%$. b) FEV1: 48\% pred; emphysema index: $18.3 \%$; BAL recovery: $7 \%$. 


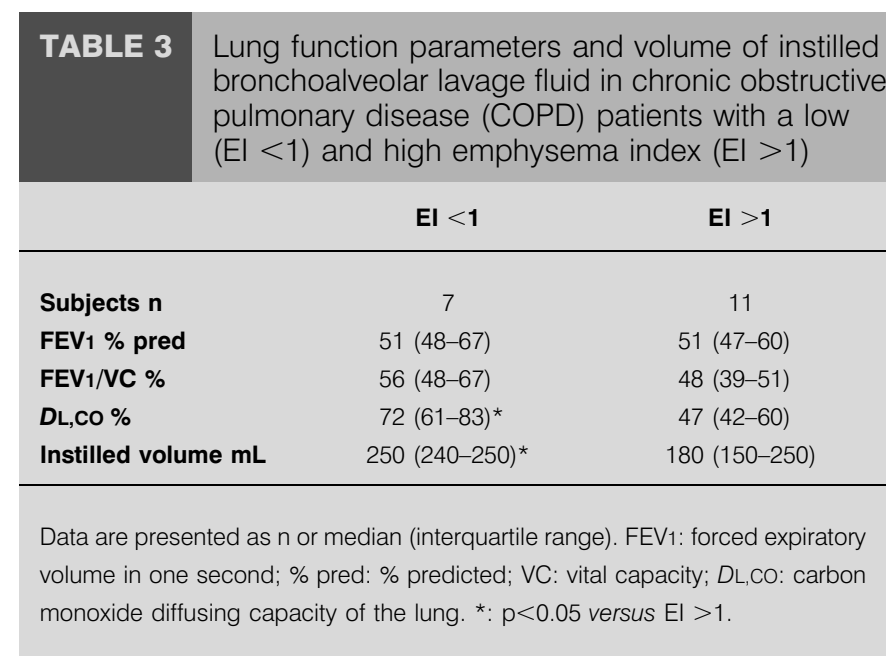

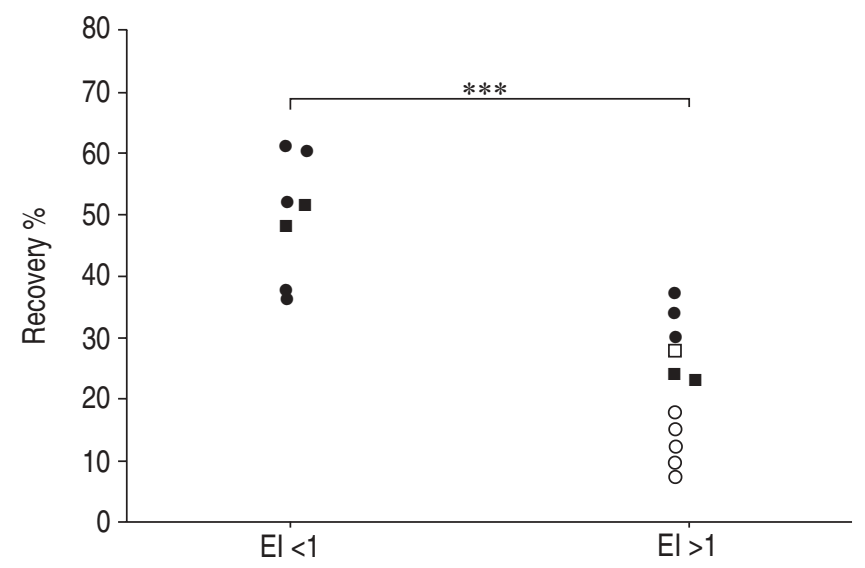

FIGURE 4. Bronchoalveolar lavage (BAL) recovery as a percentage of the instilled fluid in chronic obstructive pulmonary disease patients with a low emphysema index $(E I<1)$ and a high emphysema index $(E I>1)$. $\bullet$ : patient with a BAL of $250 \mathrm{~mL}$ instilled volume; $O$ : patient with a BAL interrupted prematurely due to a low recovery; $\mathbf{a}$ : patient with a BAL interrupted due to coughing or desaturation; $\square$ : patient had a BAL of $250 \mathrm{~mL}$ performed in the right lower lobe (whereas in all the other patients BAL was performed in the middle lobe). Statistical comparison between groups used the Mann-Whitney U-test with continuity correction.

was $23 \%$ (IQR 12-30). This difference was statistically significant $(\mathrm{p}<0.001$; fig. 4). As shown in figure 4 , all patients that had their BAL interrupted due to low recovery $(n=5)$ had an EI $<1$. Of the five patients in whom the BAL was interrupted due to coughing or desaturation, two had EI $<1$ and two had EI $>1$. One of these five patients did not undergo CT examination.

BAL recovery showed a negative Spearman rank correlation to emphysema index, both expressed as a percentage of instilled volume $(\mathrm{r}=-0.72, \mathrm{p}<0.001$; fig. 5$)$ but also as absolute volume $(\mathrm{r}=-0.71, \mathrm{p}<0.001$; data not shown).

Correlations between BAL recovery and lung function tests Recovery, measured as proportion of instilled volume (\%) correlated to DL,CO (\% pred) $(\mathrm{r}=0.67, \mathrm{p}<0.05$; fig. 6$)$ and

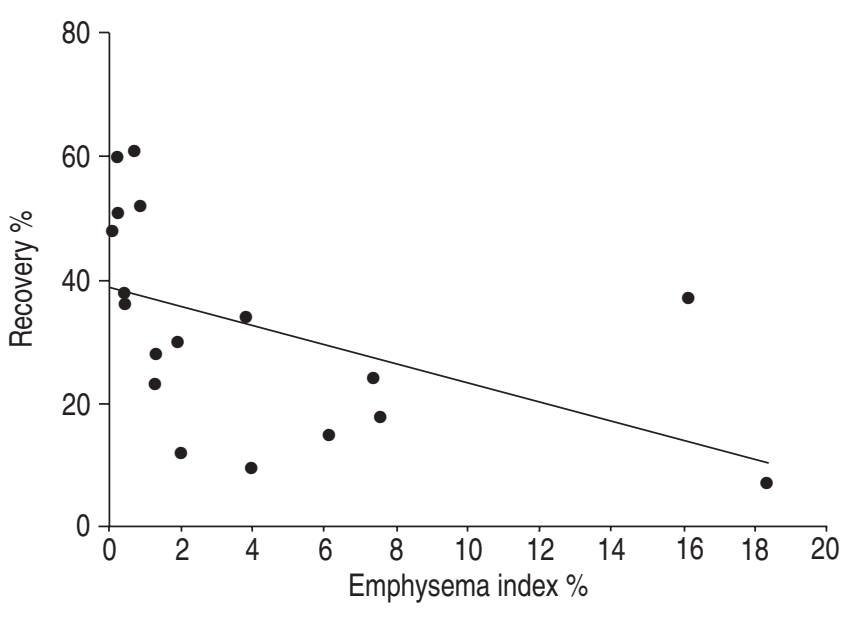

FIGURE 5. Bronchoalveolar lavage fluid recovery correlates to emphysema index; Spearman rank correlation $(r=-0.71, p<0.001)$.

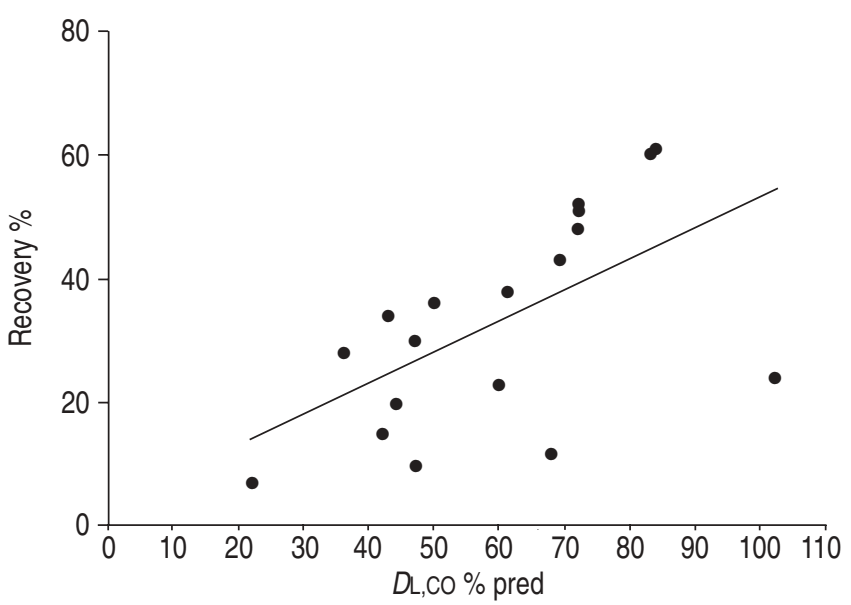

FIGURE 6. Bronchoalveolar lavage fluid recovery correlates to the carbon monoxide diffusing capacity of the lung $(D\llcorner, C O)$; Spearman rank correlation coefficient $(r=0.67, p<0.001)$.

FEV1/VC ( $r=0.52, p<0.05$; data not shown). The prognostic power of the different variables was compared by use of stepwise regression analysis. The emphysema index had a higher prognostic power than both $\mathrm{DL}, \mathrm{CO}$ and FEV1/VC. There was no correlation between recovery and the degree of airways obstruction measured as FEV1 \% pred or the degree of reversibility. Neither did recovery correlate to any of the plethysmograph data, such as total lung capacity or residual volume.

\section{DISCUSSION}

In the present study, the authors questioned whether measures of emphysema might predict BAL fluid recovery in a group of patients with moderate-to-severe COPD. COPD patients were found to have significantly lower BAL recovery than both age matched nonobstructed smokers and healthy nonsmokers. The present authors also found that within the COPD group, patients with a low emphysema index on CT scan had 
significantly higher BAL recovery. In addition, recovery correlated to DL,CO and FEV1/VC.

BAL is a useful research tool for sampling inflammatory cells from the lower respiratory tract. Since the major sites for airflow limitation in COPD are in peripheral airways and parenchyma, BAL is of particular interest in COPD research [9, $13,24]$. In previous BAL studies on COPD patients, the recovered volumes have been reported to be significantly lower in COPD patients compared with smoking control subjects with normal lung function [10, 11]. A low BAL recovery can be difficult to interpret, as it may reflect larger airways rather than the alveolar compartment. Consequently, for practical and ethical reasons, there is a need for a method to predict a high proportion of valid BAL fluid returns prior to including a group of COPD patients into a clinical study. In addition, when presenting the BAL studies of COPD patients, it is important to account for the clinical phenotype of the patients. In this context, the current authors believe that characterisation of the degree of emphysema is essential. A description of patients solely regarding the degree of airways obstruction measured as spirometry values may not be sufficient, since these parameters do not necessarily predict the BAL fluid return within a group of COPD patients.

The problem of reduced BAL recovery in COPD patients has been addressed in previous reports [9, 13, 24]. Although various causes of variability in BAL recovery have been identified [13], previous reports have not focused on the degree of emphysema in COPD.

The ERS task force on BAL has recommended that a standardised volume of lavage fluid, and a standardised number of input aliquots, should be used. However, it must be accepted that these standards will not be achievable in all subjects due to clinical constraints [13]. As expected, the BAL procedure had to be interrupted in some patients due to a low volume recovered. In these five patients, the emphysema index was $>1$. In addition, a further four patients had their BAL interrupted due to uncontrolled coughing or desaturation; these four patients were distributed equally between the two groups. In the present study, the degree of emphysema was correlated with the recovery, measured as a proportion of instilled volume (\%), which compensates reasonably for the variability in instilled volume. However, a prematurely interrupted BAL could, per se, contribute to a lower recovered volume, since the recovered proportion in aliquot three to five is higher than in aliquot one and two [12].

The difference in BAL recovery between low and high emphysema index groups showed a $0.1 \%$ significance level. The Spearman correlations between BAL recovery and $D \mathrm{~L}, \mathrm{CO}$ and $\mathrm{FEV} 1 / \mathrm{VC}$ showed significance levels at $1 \%$ or $5 \%$. Consequently, it may be possible to predict BAL recovery for a group of COPD patients, e.g. selected for a specific study, whereas the prediction of BAL recovery in a single patient is less reliable due to a wide variation of the values.

Emphysema index from examination with CT is a validated quantitative method to noninvasively assess the extent of emphysema in COPD patients [14-16]. The emphysema index correlates to histopathological specimens. However, there is no standardisation regarding parameters such as slice thickness and threshold value for "positive" pixels. In addition, differences in CT hardware and software make it difficult to compare the results from one study to another. For the same reasons, a general threshold value for dividing COPD patients into low and high emphysema index groups cannot be established. In this context, the current authors underline the importance of relating the threshold value to an experienced radiologist's evaluation of the CT scans. Due to a relatively high radiation dose from $\mathrm{CT}$ scan examinations, ethical considerations made it impossible to include a healthy nonsmoker control group.

In the present study, the mean emphysema index of the right lung CT slices was used to approximate the extent of emphysema in the right middle lobe (where the BAL was performed). However, in some emphysematous patients the disease may be heterogeneously distributed. Consequently, in these patients the mean emphysema index of CT slices from the right lung may differ from the emphysema index calculated exclusively in the middle lobe. At visual evaluation of the CT examinations, the distribution of emphysema was markedly heterogeneous in one patient. In a further two patients the distribution was intermediately heterogeneous. The remaining seven emphysematous patients were classified as having homogeneously distributed disease. Thus, an emphysema index assessment calculated exclusively in the middle lobe may have a higher prognostic value than the mean emphysema index of CT slices calculated in the whole right lung. In the present study, the mean value of the right lung was used. This provides an automatic and objective EI assessment that is easy to use, avoiding complicated manual calculations.

Airways obstruction in COPD is caused both by brochiolitis [5, 8] and emphysema [6]. The extent of emphysema can vary considerably in COPD patients with the same degree of airways obstruction. Loss of lung elastic recoil, and accompanying increased compliance, are regarded as the pathophysiological characteristics of pulmonary emphysema $[25,26]$. In the BAL procedure, fluid is suctioned back with a negative pressure connected to the working channel of the bronchoscope. The current authors believe that low BAL recovery in emphysematous patients is due to an increased compliance in bronchial walls, making them collapse as negative pressure is connected to the bronchoscope during the BAL procedure. In some patients with COPD, airway obstruction is predominantly due to bronchiolitis. This may explain the lack of correlation between BAL recovery and FEV1.

In conclusion, the presented results indicate that the extent of emphysema (measured as an emphysema index and the carbon monoxide diffusing capacity of the lung) may predict a low bronchoalveolar lavage recovery in patients with moderate-to-severe chronic obstructive pulmonary disease. Emphysema index and the carbon monoxide diffusing capacity of the lung may, therefore, be useful parameters when chronic obstructive pulmonary disease patients are selected for bronchoscopy and bronchoalveolar lavage. The present study underlines the importance of phenotyping 
chronic obstructive pulmonary disease patients regarding the extent of emphysema.

\section{ACKNOWLEDGEMENTS}

The authors would like to acknowledge H. Blomqvist, M. Dahl, B. Dahlberg and G. de Forest for technical assistance. The authors would also like to thank P. Näsman for assistance regarding the statistical methods.

\section{REFERENCES}

1 Jeffery PK. Remodeling in asthma and chronic obstructive lung disease. Am J Respir Crit Care Med 2001; 164: Suppl. 10, S28-S38.

2 Saetta M, Turato G. Airway pathology in asthma. Eur Respir J 2001; 18: Suppl. 34, 18s-23s.

3 O'Shaughnessy TC, Ansari TW, Barnes NC, Jeffery PK. Inflammation in bronchial biopsies of subjects with chronic bronchitis: inverse relationship of CD8+ T lymphocytes with FEV1. Am J Respir Crit Care Med 1997; 155: 852-857.

4 Turato G, Zuin R, Miniati M, et al. Airway inflammation in severe chronic obstructive pulmonary disease: relationship with lung function and radiologic emphysema. Am J Respir Crit Care Med 2002; 166: 105-110.

5 Hogg JC, Macklem PT, Thurlbeck WM. Site and nature of airway obstruction in chronic obstructive lung disease. N Engl J Med 1968; 278: 1355-1360.

6 Wright JL, Cagle P, Churg A, Colby TV, Myers J. Diseases of the small airways. Am Rev Respir Dis 1992; 146: 240-262.

7 Shaw RJ, Djukanovic R, Tashkin DP, Millar AB, du Bois RM, Orr PA. The role of small airways in lung disease. Respir Med 2002; 96: 67-80.

8 McLean A, Warren PM, Gillooly M, MacNee W, Lamb D. Microscopic and macroscopic measurements of emphysema: relation to carbon monoxide gas transfer. Thorax 1992; 47: 144-149.

9 Klech $\mathrm{H}$, Hutter $\mathrm{C}$, eds. Clinical guidelines and indications for bronchoalveolar lavage (BAL): Report of the European Society of Pneumology Task Group on BAL. Eur Respir J 1990; 3: 937-974.

10 Linden M, Rasmussen JB, Piitulainen E, et al. Airway inflammation in smokers with nonobstructive and obstructive chronic bronchitis. Am Rev Respir Dis 1993; 148: 1226-1232.

11 Soler N, Ewig S, Torres A, Filella X, Gonzalez J, Zaubet A. Airway inflammation and bronchial microbial patterns in patients with stable chronic obstructive pulmonary disease. Eur Respir J 1999; 14: 1015-1022.

12 Martin TR, Raghu G, Maunder RJ, Springmeyer SC. The effects of chronic bronchitis and chronic air-flow obstruction on lung cell populations recovered by bronchoalveolar lavage. Am Rev Respir Dis 1985; 132: 254-260.

13 Haslam P, Baughman R, eds. Report of European Respiratory Society (ERS) Task Force: Guidelines for measurement of acellular components and recommendations for standardization of bronchoalveolar lavage (BAL). Eur Respir Rev 1999; 9: 25-157.
14 Gevenois PA, de Maertelaer V, De Vuyst P, Zanen J, Yernault JC. Comparison of computed density and macroscopic morphometry in pulmonary emphysema. Am J Respir Crit Care Med 1995; 152: 653-657.

15 Gould GA, MacNee W, McLean A, et al. CT measurements of lung density in life can quantitate distal airspace enlargement - an essential defining feature of human emphysema. Am Rev Respir Dis 1988; 137: 380-392.

16 Muller NL, Staples CA, Miller RR, Abboud RT. “Density mask". An objective method to quantitate emphysema using computed tomography. Chest 1988; 94: 782-787.

17 Kalender WA, Fichte H, Bautz W, Skalej M. Semiautomatic evaluation procedures for quantitative CT of the lung. J Comput Assist Tomogr 1991; 15: 248-255.

18 Quanjer PH. Standardized lung function testing. Report working party standardization lung function tests. European Community for Coal and Steel. Bull Europ Physiopath Respir 1983; 19: 1-95.

19 Quanjer PH, Tammeling GJ, Cotes JE, et al. Symbols, abbreviations and units. Working Party Standardization of Lung Function Tests, European Community for Steel and Coal. Eur Respir J 1993; 6: Suppl. 16, 85-100.

20 Quanjer PH, Tammeling GJ, Cotes JE, Pedersen OF, Peslin R, Yernault JC. Lung volumes and forced ventilatory flows. Report Working Party Standardization of Lung Function Tests, European Community for Steel and Coal. Official Statement of the European Respiratory Society. Eur Respir J 1993; 6: Suppl. 16, 5-40.

21 Stocks J, Quanjer PH. Reference values for residual volume, functional residual capacity and total lung capacity. ATS Workshop on Lung Volume Measurements. Official Statement of The European Respiratory Society. Eur Respir J 1995; 8: 492-506.

22 Cotes JE, Chinn DJ, Quanjer PH, Roca J, Yernault JC. Standardization of the measurement of transfer factor (diffusing capacity). Report Working Party Standardization of Lung Function Tests, European Community for Steel and Coal. Official Statement of the European Respiratory Society. Eur Respir J 1993; 6: Suppl. 16, 41-52.

23 Park SS, Janis M, Shim CS, Williams MH Jr. Relationship of bronchitis and emphysema to altered pulmonary function. Am Rev Respir Dis 1970; 102: 927-936.

24 Klech $\mathrm{H}$, Hutter C, Costabel U, eds. Clinical guidelines and indications for bronchoalveolar lavage (BAL): Report of the European Society of Pneumology Task Group on BAL. Eur Respir Rev 1992; 2: 125-127.

25 Pare PD, Brooks LA, Bates J, et al. Exponential analysis of the lung pressure-volume curve as a predictor of pulmonary emphysema. Am Rev Respir Dis 1982; 126: 54-61.

26 Osborne S, Hogg JC, Wright JL, Coppin C, Pare PD. Exponential analysis of the pressure-volume curve. Correlation with mean linear intercept and emphysema in human lungs. Am Rev Respir Dis 1988; 137: 10831088 . 